International Mathematical Forum, 2, 2007, no. 38, 1869 - 1875

\title{
A Note on Binomial Approximation for Dependent Indicators
}

\author{
K. Teerapabolarn \\ Department of Mathematics, Faculty of Science \\ Burapha University, Chonburi 20131, Thailand \\ kanint@buu.ac.th
}

\begin{abstract}
A binomial approximation theorem and its applications for dependent and identically indicators introduced by Soon [6] do not cover the case of the case of locally dependent indicators. In this paper, we further develop such an approximation theorem and give three examples to illustrate applications for this case.
\end{abstract}

\section{Mathematics Subject Classification: Primary 60F05}

Keywords: Binomial approximation, local dependence, random indicators

\section{Introduction and theorem}

Let $\Gamma$ denote an arbitrary finite index set and $|\Gamma|$ denote the number of all elements in $\Gamma$. Let $\left\{X_{\alpha}, \alpha \in \Gamma\right\}$ be random indicators with $P\left(X_{\alpha}=\right.$ $1)=1-P\left(X_{\alpha}=0\right)=p_{\alpha}$ which are not necessarily independent or identical, and let $W=\sum_{\alpha \in \Gamma} X_{\alpha}$. For $\Gamma=\{1, \ldots, n\}, \Gamma_{\alpha}=\Gamma \backslash\{\alpha\}$ and $C_{n^{\prime} p^{\prime}}=\{1-$ $\left.\left(p^{\prime}\right)^{n^{\prime}+1}-\left(1-p^{\prime}\right)^{n^{\prime}+1}\right\}\left\{\left(n^{\prime}+1\right) p^{\prime}\left(1-p^{\prime}\right)\right\}^{-1}$ where $n^{\prime}$ is the integral part of $\left\{\left(\sum_{\alpha=1}^{n} p_{\alpha}\right)^{2} / \sum_{\alpha=1}^{n} p_{\alpha}^{2}+1 / 2\right\}$ and $p^{\prime}=\sum_{\alpha=1}^{n} p_{\alpha} / n^{\prime}$. In 1996, Soon [6] gave a bound of binomial approximation to the distribution of $W$, denote by $\mathcal{L}(W)$, using Stein's method and coupling in terms of the total variation distance as in the following lemma, which is due to theorem 1 in Soon [6].

Lemma 1. For each $\alpha \in \Gamma$, let the random indicators $X_{\alpha}, X_{\beta}, J_{\beta \alpha} ; \beta \in \Gamma_{\alpha}$ be defined on the same probability space with

$$
\mathcal{L}\left(J_{\beta \alpha} ; \beta \in \Gamma_{\alpha}\right)=\mathcal{L}\left(X_{\beta} ; \beta \in \Gamma_{\alpha} \mid X_{\alpha}=1\right) .
$$




$$
\begin{gathered}
\text { Let } W_{\alpha}=\sum_{\beta \neq \alpha} X_{\beta}, W_{\alpha \beta}=\sum_{\gamma \neq \alpha, \beta} X_{\gamma}, V_{\alpha}=\sum_{\beta \neq \alpha} J_{\beta \alpha}, V_{\alpha \beta}^{\alpha}=\sum_{\gamma \neq \alpha, \beta} J_{\gamma \alpha} \text {. Then } \\
\begin{array}{c}
d_{T V}\left(\mathcal{L}(W), B\left(n^{\prime}, p^{\prime}\right)\right) \leq C_{n^{\prime} p^{\prime}}\left\{2\left(\lambda_{3}-\lambda_{2}^{2} / \lambda\right)+\lambda\left|p^{\prime}-p^{*}\right|+\sum_{\alpha} p_{\alpha} E\left|W_{\alpha}-V_{\alpha}\right|\right. \\
+2 \lambda^{-1} \sum_{\alpha, \beta}\left|p_{\alpha}-p_{\beta}\right| p_{\alpha} p_{\beta} E\left|V_{\alpha \beta}^{\alpha}-W_{\alpha \beta}\right| \\
\left.+\lambda^{-1} \sum_{\alpha, \beta}\left(p_{\alpha}-p_{\beta}\right)^{2}\left|\operatorname{Cov}\left(X_{\alpha}, X_{\beta}\right)\right|\right\}
\end{array}
\end{gathered}
$$

where $\lambda_{\alpha}=\sum_{\beta=1}^{n} p_{\beta}^{\alpha}, \lambda=\lambda_{1}$ and $p^{*}=\lambda_{2} / \lambda$.

If the random indicators $\left\{X_{\beta} ; \beta \in \Gamma\right\}$ are identical then (1.1) becomes

$$
d_{T V}\left(\mathcal{L}(W), B\left(n^{\prime}, p^{\prime}\right)\right)=d_{T V}(\mathcal{L}(W), B(n, p)) \leq C_{n p} \sum_{\alpha} p_{\alpha} E\left|W_{\alpha}-V_{\alpha}\right|
$$

These formulas do not hold when the dependence between $X_{\beta}$ 's is local. The aim of this paper is to improve the binomial approximation result in (1.2) for a sum of locally dependent random indicators.

Suppose that for each $\alpha \in \Gamma$, the subset $B_{\alpha} \subsetneq \Gamma$ with $\alpha \in B_{\alpha}$ is chosen as a neighborhood set of $\alpha$ such that $X_{\alpha}$ is independent of $\left\{X_{\beta} ; \beta \notin B_{\alpha}\right\}$. Note that

$$
p_{\alpha} E\left(J_{\beta \alpha}\right)=P\left(X_{\alpha}=1\right) E\left(X_{\beta} \mid X_{\alpha}=1\right)=E\left[E\left(X_{\alpha} X_{\beta} \mid X_{\alpha}\right)\right]=E\left(X_{\alpha} X_{\beta}\right),
$$

and by (1.2) we have

$$
\begin{aligned}
d_{T V}(\mathcal{L}(W), B(n, p)) & \leq C_{n p} \sum_{\alpha} \sum_{\beta \neq \alpha} p_{\alpha} E\left|X_{\beta}-J_{\beta \alpha}\right| \\
& =C_{n p} \sum_{\alpha \in \Gamma} \sum_{\beta \in B_{\alpha} \backslash\{\alpha\}} p_{\alpha} E\left|X_{\beta}-J_{\beta \alpha}\right| \\
& \leq C_{n p} \sum_{\alpha \in \Gamma} \sum_{\beta \in B_{\alpha} \backslash\{\alpha\}}\left\{p_{\alpha} p_{\beta}+E\left(X_{\alpha} X_{\beta}\right)\right\}
\end{aligned}
$$

The following theorem is an immediate consequence of (1.3).

Theorem 1. For arbitrary finite index set $\Gamma$, let the random variables $\left\{X_{\beta} ; \beta \in \Gamma\right\}$ be identical. Then, for all $p_{\alpha}=p$, the following formula hold:

$$
d_{T V}(\mathcal{L}(W), B(|\Gamma|, p)) \leq C_{|\Gamma| p}\left\{|\Gamma|\left(\left|B_{\alpha}\right|-1\right) p^{2}+\sum_{\alpha \in \Gamma} \sum_{\beta \in B_{\alpha} \backslash\{\alpha\}} E\left(X_{\alpha} X_{\beta}\right)\right\}
$$




\section{Applications}

This section gives three examples to illustrate applications of the theorem.

Example 1. (The birthday problem)

Suppose $n$ balls (people) are uniformly and independently distributed into $d$ boxes (days of the year). The birthday problem involves finding the approximate distribution of the number of boxes that receive $k$ or more balls for some fixed positive integer $k$. To get started, let the index set $\Gamma$ be the collection of all sets of trials $\alpha \subset\{1,2, \ldots, n\}$ having $|\alpha|=k$ elements, where $\{1,2, \ldots, n\}$ is a set of $n$ balls. Let $X_{\alpha}$ be the indicator of the event that the balls indexed by $\alpha$ all fall into the same box with probability $p_{\alpha}=P\left(X_{\alpha}=1\right)=d^{1-k}$. The number of sets of $k$ balls that fall into the same box is given by $W=\sum_{\alpha \in \Gamma} X_{\alpha}$. Since all $p_{\alpha}$ are identical, denoted by $p$, we have

$$
|\Gamma| p=\left(\begin{array}{l}
n \\
k
\end{array}\right) d^{1-k} .
$$

Following Arratia, Goldstein and Gordon [1], we can define the neighborhood $B_{\alpha}$ for $\alpha$ so that $X_{\alpha}$ is independent of those $X_{\beta}$ with $\beta$ outside $B_{\alpha}$ by taking $B_{\alpha}=\{\beta \in \Gamma: \alpha \cap \beta \neq \varnothing\}$. We observe that $X_{\alpha}$ and $X_{\beta}$ are independent if $\alpha \cap \beta=\varnothing$. For a given $\alpha, 1 \leq|\alpha \cap \beta| \leq k-1$ for $\beta \in B_{\alpha} \backslash\{\alpha\}$, we obtain

$$
\begin{aligned}
\sum_{\alpha \in \Gamma} \sum_{\beta \in B_{\alpha} \backslash\{\alpha\}} E\left(X_{\alpha} X_{\beta}\right) & =\left(\begin{array}{l}
n \\
k
\end{array}\right) \sum_{j=1}^{k-1}\left(\begin{array}{l}
k \\
j
\end{array}\right)\left(\begin{array}{l}
n-k \\
k-j
\end{array}\right) d^{1+j-2 k} \\
& =|\Gamma| p b,
\end{aligned}
$$

where $b=\sum_{j=1}^{k-1}\left(\begin{array}{l}k \\ j\end{array}\right)\left(\begin{array}{l}n-k \\ k-j\end{array}\right) d^{j-k}$.

When applying the theorem, we have

$$
d_{T V}(\mathcal{L}(W), B(|\Gamma|, p)) \leq C_{|\Gamma| p}|\Gamma| p\left\{\left(\left|B_{\alpha}\right|-1\right) p+b\right\}
$$

where $\left|B_{\alpha}\right|=\left(\begin{array}{l}n \\ k\end{array}\right)-\left(\begin{array}{c}n-k \\ k\end{array}\right)$.

If $k=2, n=5$ and $d=30$, then we have $|\Gamma| p=\left(\begin{array}{l}5 \\ 2\end{array}\right)(30)^{-1}=1 / 3,\left|B_{\alpha}\right|=$ $\left(\begin{array}{l}5 \\ 2\end{array}\right)-\left(\begin{array}{l}3 \\ 2\end{array}\right)=7$ and $b=2\left(\begin{array}{l}3 \\ 1\end{array}\right)(30)^{-1}=0.20$. Hence, by $(2.1)$, a bound for approximating the distribution of the number of sets of three balls that fall into the same box by binomial distribution is

$$
d_{T V}\left(\mathcal{L}(W), B\left(10,30^{-1}\right)\right) \leq 0.11709504 .
$$


This shows the approximation above has an error of no more than 0.11709504, which is less than the bound, 0.12283643 , on Poisson approximation using the result of Corollary 2.C.5 in Barbour, Holst and Janson [2].

Example 2. (A random graph problem)

Consider a graph with $n$ nodes which is created by randomly connecting some pairs of nodes by edges. If the connection probability per pair is $p$, then all pairs from a triple of nodes are connected with probability $p^{3}$. Let $\Gamma$ be the set of all triple of nodes in the random graph, and let $W$ be the number of such triangles in a random graph. So $W=\sum_{\alpha \in \Gamma} X_{\alpha}$ where $X_{\alpha}=1$ if triple of nodes $\alpha$ is connected to be the triangle and $X_{\alpha}=0$ otherwise. We then have $p_{\alpha}=P\left(X_{\alpha}=1\right)=p^{3}$.

Now we apply the theorem for bounding the error of this approximation by taking $B_{\alpha}=\{\beta:|\alpha \cap \beta| \geq 2\}$ so that $X_{\alpha}$ and $X_{\beta}$ are independent for $\beta \notin B_{\alpha}$. For $\alpha \neq \beta, E\left(X_{\alpha} X_{\beta}\right)=p^{5}$ and $\left|B_{\alpha}\right|=3(n-3)+1$. Hence, by (1.4), we have

$$
d_{T V}\left(\mathcal{L}(W), B\left(|\Gamma|, p^{3}\right)\right) \leq C_{|\Gamma| p^{3}} 3(n-3)|\Gamma| p^{5}(1+p) .
$$

For $n=10$ and $p=0.15$, we get $|\Gamma|=\left(\begin{array}{c}n \\ 3\end{array}\right)=\left(\begin{array}{c}10 \\ 3\end{array}\right)=120$ and a bound of the error for approximating the distribution of the number of triangles in a random graph by the binomial distribution with parameter $|\Gamma|=120$ and $p^{3}=0.003375$, following $(2.2)$, is

$$
d_{T V}(\mathcal{L}(W), B(120,0.003375)) \leq 0.18153197
$$

It is less than a bound on Poisson approximation as the following result.

$$
\begin{aligned}
d_{T V}(\mathcal{L}(W), P o(0.405)) & \leq\left(1-e^{-0.405}\right)\left\{3(n-3) p^{2}(1+p)+p^{3}\right\} \\
& =0.18208043 .
\end{aligned}
$$

Example 3. (The somatic cell hybrids)

Somatic cell hybrids are routinely used to assign particular human genes to particular chromosomes [3,7] and are constructed by fusing normal human cells with permanently transformed mouse cells. The resulting hybrid cells retain all of the mouse chromosomes while losing random subsets of the human chromosomes. It is possible to establish a correspondence between the presence or absence of a given human gene and the presence or absence of each of the 24 distinct human chromosomes in each clone and one can assign the gene to a particular chromosome. The information of a panel of somatic cell can be found in Goradia and Lange [4] and Lange [5].

Let $n$ denote the number of hybrid clones in a panel. Since hybrids are usually created from human female cells. This give 22 autosomes and the X chromosome. Fig. 1 shows hybrid panel with $n=9$ clones. Each row 
of this panel corresponds to a particular clone, and each of the 23 columns corresponds to a particular chromosome. A 1 in row $\mathrm{i}$ and column $\mathrm{j}$ of the panel indicates the presence of chromosome $\mathrm{j}$ in clone $\mathrm{i}$. A 0 indicates the absence of a chromosome in a clone. In creating a test column for a human gene, a test column of 0's and 1's is constructed when each clone is assayed for a presence of a given human gene.

$$
\begin{array}{lllllllllllllllllllllll}
0 & 1 & 0 & 1 & 0 & 0 & 0 & 1 & 0 & 0 & 0 & 0 & 0 & 0 & 1 & 0 & 1 & 1 & 0 & 1 & 1 & 1 & 1 \\
1 & 0 & 1 & 0 & 1 & 1 & 0 & 0 & 1 & 0 & 0 & 0 & 0 & 1 & 0 & 0 & 1 & 0 & 1 & 0 & 1 & 1 & 1 \\
0 & 1 & 1 & 1 & 1 & 0 & 1 & 0 & 0 & 0 & 0 & 0 & 1 & 0 & 0 & 1 & 1 & 0 & 1 & 1 & 0 & 1 & 1 \\
1 & 1 & 1 & 0 & 0 & 1 & 1 & 0 & 0 & 1 & 0 & 1 & 0 & 0 & 0 & 1 & 1 & 1 & 0 & 0 & 1 & 0 & 1 \\
0 & 0 & 0 & 1 & 1 & 1 & 1 & 0 & 0 & 0 & 1 & 1 & 1 & 1 & 1 & 0 & 1 & 0 & 0 & 0 & 1 & 1 & 0 \\
0 & 1 & 1 & 1 & 1 & 1 & 1 & 1 & 1 & 1 & 1 & 0 & 0 & 0 & 0 & 0 & 1 & 0 & 0 & 0 & 0 & 0 & 0 \\
0 & 0 & 1 & 0 & 1 & 0 & 1 & 1 & 0 & 1 & 1 & 1 & 0 & 0 & 0 & 0 & 1 & 1 & 1 & 1 & 1 & 0 & 0 \\
0 & 0 & 0 & 1 & 0 & 1 & 1 & 1 & 0 & 0 & 0 & 1 & 0 & 1 & 1 & 1 & 1 & 0 & 1 & 0 & 1 & 0 & 1 \\
1 & 0 & 0 & 0 & 1 & 1 & 0 & 0 & 0 & 1 & 0 & 1 & 1 & 0 & 1 & 0 & 1 & 0 & 1 & 1 & 0 & 0 & 1
\end{array}
$$

Fig. 1. A Somatic Cell Hybrid Panel

The panel in Fig. 1 has the unusual property that every pair of column differs in at least three entries. This level of redundancy is useful for assigning the gene to a particular chromosome, such as if a single assay error is made in constructing a test column for a human gene, then the gene can be successfully assigned to a particular human chromosome. In practice the chromosome constitution of a clone cannot be predicted in advance, and the level of redundancy is random. Minimum Hamming distance is a natural measure of the redundancy of a panel. The Hamming distance $\rho\left(c_{s}, c_{t}\right)$ between two columns $c_{s}$ and $c_{t}$ from a random panel is just the number of entries in which they differ.

Let $\Gamma=\{\alpha=\{s, t\}: 1 \leq s \neq t \leq 23\}$ be an index set of pairs of chromosomes, then the minimum Hamming distance of a panel is defined as $\min _{\{s, t\} \in \Gamma} \rho\left(C_{s}^{n}, C_{t}^{n}\right)$ where $C_{s}^{n}$ denote the column $s$ of a random panel of $n$ clones. For any two columns, $C_{s}^{n}$ and $C_{t}^{n}, X_{\{s, t\}}$ is defined to be the indicator of the event the Hamming distance, $\rho\left(C_{s}^{n}, C_{t}^{n}\right)$, is less than some fixed Hamming distance $d$. For each $\alpha \in \Gamma$, the probability $P(X=1)$ is

$$
p=\sum_{i=0}^{d-1}\left(\begin{array}{c}
n \\
i
\end{array}\right) q^{i}(1-q)^{n-i}
$$

where $q=2 p_{0}\left(1-p_{0}\right)$ is the probability that $C_{s}^{n}$ and $C_{t}^{n}$ differ in any entry and $p_{0}$ is a retention probability applying to all chromosome pairs. 
Let $W=\sum_{\alpha \in \Gamma} X_{\alpha}$ be the number of pairs of chromosomes for which the Hamming distance, $\rho\left(C_{s}^{n}, C_{t}^{n}\right)$, is less than some fixed Hamming distance $d$. If $W=0$, then the minimum Hamming distance equals or exceeds d. It can be approximated the distribution of $W$ by a binomial distribution with parameter $|\Gamma|$ and $p,|\Gamma|=\left(\begin{array}{c}23 \\ 2\end{array}\right)=253$.

For each $\alpha \in \Gamma$, we set $B_{\alpha}=\{\beta \in \Gamma: \alpha \cap \beta \neq \varnothing\}$ as a neighborhood of dependence for $\alpha$. It is observe that $X_{\alpha}$ is independent of the entire $X_{\beta}$ for $\beta \notin B_{\alpha}$. Since $\left|B_{\alpha}\right|=\left(\begin{array}{c}23 \\ 2\end{array}\right)-\left(\begin{array}{c}21 \\ 2\end{array}\right)=43$ and $E\left(X_{\alpha}, X_{\beta}\right)$ does not depend on the particular column pair $\beta \in B_{\alpha} \backslash\{\alpha\}$ chosen (Lange [5, p.257]). Thus ,by (1.4), a bound for approximating the distribution of $W$ is of the form

$$
d_{T V}(\mathcal{L}(W), B(|\Gamma|, p)) \leq 10626 C_{|\Gamma| p}\left\{p^{2}+E\left(X_{\alpha}, X_{\beta}\right)\right\}
$$

In the case of $p_{0}=0.50$, it follows form Lange [5, p.257] that $X_{\alpha}$ and $X_{\beta}$ are independent for each $\alpha \in \Gamma$ and $\beta \in B_{\alpha} \backslash\{\alpha\}$ which lead to $E\left(X_{\alpha}, X_{\beta}\right)=p^{2}$. If $p_{0}=0.50, d=3$ and $n=20$, then $p=0.000201225$ and a bound for approximating the distribution of the number of pairs of chromosomes for which the Hamming distance is less than 3 is

$$
d_{T V}(\mathcal{L}(W), B(253,0.000201225)) \leq 0.00083916
$$

and the probability approximation of the Hamming distance equals or exceeds 3 is

$$
0.94952018 \leq P(W=0) \leq 0.95119849
$$

Table 1. Binomial estimates of $P(W>0)$

\begin{tabular}{ccccc}
\hline$d$ & $n$ & Estimate & Lower Bound & Upper Bound \\
\hline 1 & 10 & 0.21901 & 0.20103 & 0.23698 \\
1 & 15 & 0.00769 & 0.00767 & 0.00771 \\
1 & 20 & 0.00024 & 0.00024 & 0.00024 \\
1 & 25 & 0.00001 & 0.00001 & 0.00001 \\
2 & 10 & 0.93494 & 0.08486 & 1.00000 \\
2 & 15 & 0.11624 & 0.11147 & 0.12100 \\
2 & 20 & 0.00505 & 0.00505 & 0.00506 \\
2 & 25 & 0.00020 & 0.00020 & 0.00020 \\
3 & 10 & 1.00000 & 0.00000 & 1.00000 \\
3 & 15 & 0.60779 & 0.41886 & 0.79672 \\
3 & 20 & 0.04964 & 0.04880 & 0.05048 \\
3 & 25 & 0.00246 & 0.00245 & 0.00246 \\
\hline
\end{tabular}


In the case of $W=0$, an interesting point is the probability approximation of $W>0, P(W>0)=1-P(W=0)$, and some binomial estimates of $P(W>0)$ for $p_{0}=0.50$ show in Table 1 .

It can be seen that the estimates are accurate when $n$ is increasing, i.e. the bound of error is small when $n$ is large. By comparing the lower and upper bounds, the binomial estimates of $P(W>0)$ are a little better than the Poisson estimates of Lange [5, p.257].

\section{References}

[1] R. Arratia, L. Goldstein and L. Gordon, Two moments suffice for Poisson approximations:the Chen-Stein method, Annals of probability, 17 (1989), $9-25$.

[2] A.D. Barbour, L. Holst and S. Janson, Poisson approximation, Oxford Studies in probability 2, Clarendon Press, Oxford, 1992.

[3] P. D'Eustachio and F.H. Ruddle, Somatic cell genetics and gene families, Science, 220 (1983), 919-924.

[4] T.M. Goradia and K. Lange, Applications of coding theory to the design of somatic cell hybrid panels, Mathematical Bioscience, 91 (1988), 201-219.

[5] K. Lange K, Applied Probability, Springer-Verlag, New York, 2003.

[6] Y.T. Soon Spario, Binomial approximation for dependent indicators, Statistica Sinica, 6 (1996), 703-714.

[7] M. Weiss and H. Green, Human-mouse hybrid cell lines containing partial complements of human chromosomes and functioning human gene, Proceedings of the National Academy of Sciences of the United States of America, 58 (1967), 1104-1111.

Received: December 13, 2006 